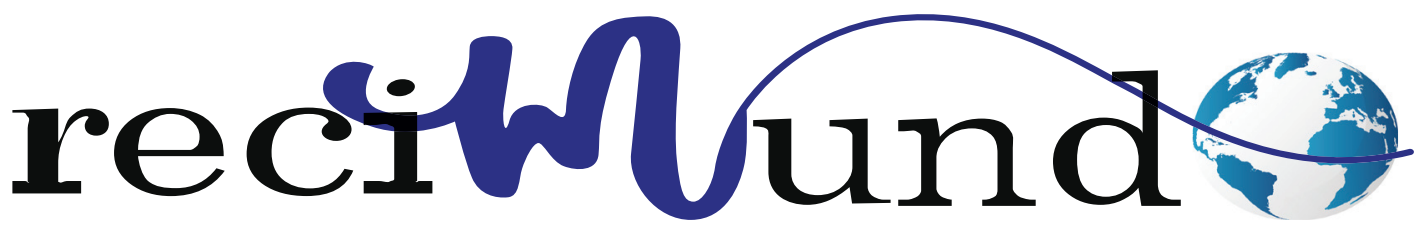

Revista Científica Mundo de la Investigación y el Conocimiento

DOl: 10.26820/recimundo/4.(1).enero.2020.403-415

URL: http://recimundo.com/index.php/es/article/view/770

EDITORIAL: Saberes del Conocimiento

REVISTA: RECIMUNDO

ISSN: 2588-073X

TIPO DE INVESTIGACIÓN: Artículo de Revisión CÓDIGO UNESCO: 3201 Ciencias Clínicas PAGINAS: $403-415$

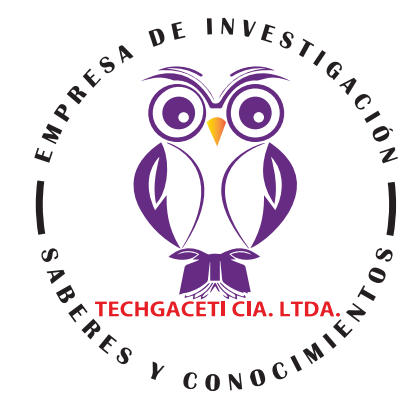

\title{
Prevención y cuidado de los pacientes diagnosticados con aneurisma cerebral
}

\section{Prevention and care of patients diagnosed with cerebral aneurysm}

\section{Prevenção e atendimento de pacientes diagnosticados com aneurisma cerebral}

Edisson Javier Santana Bailón'; Diana Julissa Cevallos Vega2; Cecilia Isabel Moyano Vega3; Richard Iván Burgos Delgado ${ }^{4}$; Yadira Xiomar Arteaga Castro5; Jonathan Paul Durán González ${ }^{6}$

\section{RECIBIDO: 18/09/2019 ACEPTADO: 29/10/2019 PUBLICADO: 31/01/2020}

1. Médico Cirujano; Investigador Independiente; Manta, Ecuador; edisonsantanab@hotmail.es; (D) https://orcid. org/0000-0002-7934-5452

2. Médica Cirujana; Hospital Rodríguez Zambrano; Manta, Ecuador; diyucevaveg@hotmail.com; (D) https://orcid. org/0000-0001-8766-2653

3. Médica Cirujana; Hospital de Especialidades Eugenio Espejo; Quito, Ecuador; cheoychabe@hotmail.com; (1) https://orcid.org/0000-0003-1757-6500

4. Médico Cirujano; Hospital Rafael Rodríguez Zambrano; Manta, Ecuador; richardburgosdelgado@gmail.com; (D) https://orcid.org/0000-0003-1188-7674

5. Médica Cirujana; Médico de Primer Nivel de Atención de Salud Distrito de Salud 13D04, Portoviejo, Ecuador; xio_marita91@hotmail.com; (D) https://orcid.org/0000-0001-7780-6377

6. Médico Cirujano; Médico Residente de Emergencia-Centro Clínico Quirúrgico Hospital del Día IESS, Jipijapa, Ecuador; jhonduran90@hotmail.com; (D) https://orcid.org/0000-0003-3735-0541

CORRESPONDENCIA

Edisson Javier Santana Bailón

edisonsantanab@hotmail.es

\section{Manta, Ecuador}




\section{RESUMEN}

Los aneurismas cerebrales son dilataciones ubicadas de la pared del vaso arterial encontrándose en puntos de bifurcación. Los aneurismas cerebrales tienen potencial de ruptura y causan complicaciones serias como accidente cerebro-vascular hemorrágico, situación clínica crítica conocida como hemorragia subaracnoidea (HSA). De allí la importancia de su detección en tiempo oportuno. Se visualizan mediante la tomografía computarizada (TC) y la Resonancia Magnética Nuclear (RMN), siendo estas, las técnicas deimagen más efectiva para detectar y evaluar los aneurismas cerebrales. La TC utiliza radiación ionizante y un medio de contraste yodado para obtener imágenes del sistema vascular cerebral; así mismo, la RMN obtiene imágenes mediante campos magnéticos y el flujo sanguíneo. Una vez se ha diagnosticado, requiere de atención inmediata dentro de la unidad de cuidados intensivos. (Carmona Simarro, Gallego Lopez, \& LLabata Carabal, 2005), una observación y valoración continúa sobre signos y síntomas a pacientes con esta patología, para evitar riesgos o complicaciones. El re-sangrado es el peligro más inminente y debe evitarse por la oclusión endovascular con coils o por el clipaje quirúrgico del aneurisma; la manifestación de isquemia cerebral diferida es disminuida con la administración de nimodipino y evitando la hipovolemia. Los especialistas utilizan opciones terapéuticas quirúrgicas y endo-vasculares, aplicación de tratamiento cuando se presentan las complicaciones mayores, tales como re-sangrado, vaso-espasmo cerebral o hidrocefalia aguda en la Unidad de Cuidados Intensivos. La prevención y el cuidado del paciente diagnosticado con aneurisma cerebral es el objeto del presente estudio en aras de restablecer las funciones vitales y prevenir complicaciones.

Palabras clave: Hemorragia Subaracnoidea, Aneurisma Intracraneal, Vasoespasmo Intracraneal, Hidrocefalia, Nimodipino, Embolización, Sacular, Fusiforme, Disecante, Clipadomicrovascular.

\section{ABSTRACT}

Cerebral aneurysms are dilations located in the wall of the arterial vessel being at bifurcation points. Cerebral aneurysms have rupture potential and cause serious complications such as hemorrhagic cerebrovascular accident, a critical clinical situation known as subarachnoid hemorrhage (SAH), hence the importance of its detection in a timely manner. They are visualized by computed tomography (CT) and Nuclear Magnetic Resonance (NMR), these being the most effective imaging techniques to detect and evaluate cerebral aneurysms. CT uses ionizing radiation and an iodinated contrast medium to obtain images of the cerebral vascular system; likewise, NMR obtains images through magnetic fields and blood flow. Once it has been diagnosed, it requires immediate attention within the intensive care unit. (Carmona Simarro, Gallego Lopez, \& L. Labata Carabal, 2005), an observation and assessment continues on signs and symptoms to patients with this pathology, to avoid risks or complications. Re-bleeding is the most imminent danger and should be avoided by endovascular occlusion with coils or by surgical clipping of the aneurysm; the manifestation of deferred cerebral ischemia is diminished with the administration of nimodipine and avoiding hypovolemia. Specialists use surgical and endovascular vascular options, treatment application when major complications occur, such as re-bleeding, cerebral vaso-spasm or acute hydrocephalus in the Intensive Care Unit. The prevention and care of the patient diagnosed with cerebral aneurysm is the object of the present study in order to restore vital functions and prevent complications.

Keywords: Subarachnoid Hemorrhage, Intracranial Aneurysm, Intracranial Vasospasm, Hydrocephalus, Nimodipine, Embolization, Sacular, Fusiform, Dissecting, Clipadomicrovascular.

\section{RESUMO}

Aneurismas cerebrais são dilatações localizadas na parede do vaso arterial em pontos de bifurcação. Os aneurismas cerebrais têm potencial de ruptura e causam sérias complicações, como acidente vascular cerebral hemorrágico, uma situação clínica crítica conhecida como hemorragia subaracnóidea (HAS), daí a importância de sua detecção em tempo hábil. Eles são visualizados por tomografia computadorizada (TC) e ressonância magnética nuclear (RMN), sendo estas as técnicas de imagem mais eficazes para detectar e avaliar aneurismas cerebrais. A TC utiliza radiação ionizante e meio de contraste iodado para obter imagens do sistema vascular cerebral; da mesma forma, a RMN obtém imagens através de campos magnéticos e fluxo sanguíneo. Uma vez diagnosticado, requer atenção imediata na unidade de terapia intensiva. (Carmona Simarro, Gallego Lopez e L. Labata Carabal, 2005), uma observação e avaliação continuam sobre sinais e sintomas para pacientes com essa patologia, para evitar riscos ou complicações. O re-sangramento é o perigo mais iminente e deve ser evitado por oclusão endovascular com serpentinas ou por recorte cirúrgico do aneurisma; a manifestação de isquemia cerebral adiada é diminuída com a administração de nimodipina e evitando a hipovolemia. Os especialistas usam opções vasculares cirúrgicas e endovasculares, aplicação de tratamento quando ocorrem complicações maiores, como re-sangramento, vaso-espasmo cerebral ou hidrocefalia aguda na Unidade de Terapia Intensiva. A prevenção e o cuidado do paciente diagnosticado com aneurisma cerebral são o objetivo do presente estudo, a fim de restabelecer funções vitais e prevenir complicações.

Palavras-chave: Hemorragia subaracnóidea, aneurisma intracraniano, vasoespasmo intracraniano, hidrocefalia, nimodipina, embolização sacular, fusiforme, dissecação, clipado microvascular. 


\section{Introudcción}

Los aneurismas cerebrales predominan en la población entre el 1 y $6 \%$. (Guidelines for the Surgical Treatment of Unruptured Intracranial Aneurysms, 2008). La hemorragia subaracnoidea (HSA) secundaria a ruptura de aneurismas cerebrales se evidencia entre 5 a $15 \%$ de los casos de enfermedad cerebrovascular,(Mocco, Zacharia, Komotar, \& ét al, 2006). En los Estados Unidos de Norteamérica la hemorragia subaracnoidea produce hasta 30000 aneurismas por año. (Harrod, Bendok, Batjer , \& ét al, 2005) (Lauric, Miller, Frisken, \& ét al, 2010).

Es importante, diagnosticar oportunamente al paciente a través estudios de neuro-imagen como la tomografía computarizada de cráneo con angiotomografía y la resonancia magnética con angioresonancia o incluso la angiografía cerebral. De igual manera, se puede identificar su morfología, tamaño y localización, determinando el riesgo de ruptura, debido a su tamaño, grupo de edad en que se presenta, demostrando enfermedades relacionadas. (Guidelines for the Surgical Treatment of Unruptured Intracranial Aneurysms, 2008). Los aneurismas sin ruptura de tamaño de 7 a $10 \mathrm{~mm}$ en población adulta hasta de 50 años demuestran caso clínico anual de hemorragia subaracnoidea de $1 \%$ incrementándose hasta $4 \%$ a partir de los 70 años. (Guidelines for the Surgical Treatment of Unruptured Intracranial Aneurysms, 2008).

Los aneurismas intracraneales son frecuentes en enfermedad poli-quística renal, la enfermedad de EhrlenDanlos, coartación de la aorta, enfermedad de Marfán,Morquio, pseudoxantomaelasticum, hiper-aldosteronismo y en pacientes con antecedentes familiares de aneurisma intracraneal (Broderick, Sauerbeck, Foroud, \& ét al, 2005).

En Latino América el $4 \%$ de la población puede tener aneurisma cerebral, el $2 \%$ se muestra durante la niñez y el $4 \%$ en las mujeres todas con probabilidad en riesgo de ruptura y sangrado, el $12 \%$ de los pacientes mueren antes de llegar al hospital, el 45\% fallece en los primeros 30 días y en el 50\% de los sobrevivientes le queda algún tipo de secuela irreversible, por lo tanto, un 60\% corresponde al sexo femenino, se dice un número aproximado de 150.000 portadores de aneurismas cerebrales, que aún no han sufrido la rotura del mismo.

Según estudios, ellnstituto Nacional de Estadísticas y Censos del Ecuador demuestran un total de 846 ingresos hospitalarios para el año 2013, al estudiar los casos por edad de los pacientes, se visualiza que ésta patología incrementa su frecuencia de aparición conforme pasan los años de las personas, así mismo, se reportan $161 \mathrm{ca}-$ sos en pacientes con edades entre 55 y 65 años. (INEC, 2015). El aneurisma cerebral aumenta con la edad, el $80 \%$ de los casos aproximadamente se producen en personas de 40-65 años, un 15\% en personas de 20 a 40 años, (INEC, 2015). El 5\% de los casos ocurren en personas menores de 20 años, Con referencia, a la organización mundial de la salud, el AC (aneurisma cerebral) constituye la tercera causa de muerte femenina $(9.7 \%)$, de las cuales 4.95 millones ocurren en países con ingresos medios y bajos. (OMS, 2015).

Los servicios de cuidados intensivos, actualmente, son aéreas de atención hospitalaria destinada a admitir pacientes en estado de gravedad con patologías que requieren de tratamiento médico - quirúrgico con probabilidad de recuperación, que exigen asistencia médica y cuidados de enfermería permanentes, con la utilización de equipos especializados. El tratamiento intensivo adopta todas las posibilidades terapéuticas para la sustitución de funciones orgánicas alteradas o suprimidas. Teniendo como objetivo restablecer las funciones vitales y recuperar valores necesarios para la continuación de la vida.

\section{Metodologia}

La revisión que se presenta en este artículo es de tipo documental, la recopilación de la 
información se ha realizado a través de una investigación con análisis bibliográfico y de estudios casos encontrados a través de medios Electrónicos, en donde hay disponible material de consulta sobre el aneurisma cerebral. Aquí se consideran los aportes más importantes de ciertos autores, con el objetivo de dejar abierta la investigación para futuros casos que den soporte a nuevos estudios.

\section{Resultado}

\section{Aneurisma cerebral}

Es una hinchazón anormal de un fragmento de la arteria intracraneal originada por la debilidad en la pared del vaso sanguíneo, afectado por la presión arterial y la hemodinamia" (FUCAC, S.F.). El aneurisma puede manifestar una rotura y causar sangrado en el cerebro. En consecuencia, la rotura se origina en la zona entre el cerebro y los tejidos delgados que reviste el mismo y se denomina hemorragia subaracnoidea (HSA) que se convierte en una amenaza para la vida y requiere el tratamiento médico inmediato. (Mayo Clinic, 2017).

Los aneurismas cerebrales pequeños no sangran ni causan otros problemas ya que se producen en cualquier lugar del cerebro, encontrándose habitualmente junto al bucle de arterias que pasan entre la parte inferior del cerebro y la base del cráneo. (National Institute of Neurological, 2016)

La desintegración de elastina provocada por la consecuencia de la aterosclerosis, el tabaco, el envejecimiento y la inflamación,siendo estos, elemento clave en la formación del aneurisma.(García \& Pardo). E crecimiento del diámetro del aneurisma cerebral produce un aumento en la tensión de la pared arterial.

Mientras que, va creciendo el aneurisma incrementa la tensión de la pared arterial permitiendo la ruptura. Se desconoce con precisión la causa, la mayoría son congénitos debido a una anormalidad innata de una pared arterial. Dicho de otro modo, son más comunes en personas con ciertas enfermedades genéticas como trastornos del tejido conjuntivo y enfermedad del riñón poliquístico. (National Institute of Neurological, 2016). Otras causas pueden ser: trauma o lesión craneal, crisis hipertensivas, infecciones, tumores, enfermedades del sistema vascular, abuso de drogas. (National Institute of Neurological, 2016).

La anomalía del tejido conectivo y de la matriz extracelular de la pared vascular. Incluye múltiples afecciones nutricionales, inflamatorias y síndromes genéticos. La deficiencia de vitamina $C$, por trastorno alimenticios genera un desequilibrio en la síntesis y degradación del colágeno que afecta la pared de los vasos sanguíneos principalmente en el contexto de enfermedades inflamatorias.

\section{Factores de Riesgo}

- Edad avanzada (45 años en adelante).

- Sexo femenino.

- Antecedentes familiares de aneurisma.

- Consumo de drogas.

- Presión arterial elevada.

\section{Clasificación de los Aneurismasde acuer- do a su morfología}

1.- Aneurisma sacular; se muestra como un bulto sobre una pared del vaso sanguíneo, son los más recurrentes formándose de manera silenciosa desconociendo aunla causa, según investigaciones, hay tendencias hereditarias a tener vasos sanguíneos débiles; se presenta mayormente en mujeres de 40 a 59 años en comparación con los hombres cuya edad oscila entre 25 y 49 años.

2.- Aneurisma fusiforme; está formado por el ensanchamiento de todas las paredes del vaso, a su vez se puedes clasificar por tamaño. Cuando un aneurisma aumenta de tamaño, puede provocar cefaleas o dolor localizado ejerciendo presión sobre el tejido 
cerebral normal o los nervios adyacentes. Al comprimirse los vasos sanguíneos generan problemas visuales, adormecimiento o debilidad en un brazo o una pierna, alteraciones de la memoria, del habla o convulsiones.

Clasificación de aneurisma fusiforme según su tamaño:

- $\quad$ Microaneurismas $\leq 3 \mathrm{~mm}$

- Pequeños 4-6 mm

- $\quad$ Medianos 7-10 mm

- $\quad$ Grandes 11-24 mm

- $\quad$ Gigantes $\geq 25 \mathrm{~mm}$

3.- Aneurisma disecante;es la inflamación de la pared de una arteria, generalmente la aorta, complicada por la disección de la capa media arterial donde puede penetrar sangre presentando riesgo de rotura.

\section{Síntomas de alarmay Cuidados prelimi- nares}

Alromperse un aneurisma cerebral, la sangre se escurre directamente hacia el cerebro, o sus envolturas, las meninges. Cuando el derrame de sangre en el cerebro es de gran cantidad, puede originar síntomas de cefalea intensa, náuseas, vómitos o somnolencia e incluso coma, provocando daño directo a las células circundantes lo que aumenta la presión dentro del cráneo, interrumpiendo el suministro de sangre y oxígeno al cerebro,cuando la presión es muy elevada la gravedad de la patología puede condicionar al paciente un alto número de secuelas neurológicas, que pudieran provocar incapacidad continua y prolongada, afectando el modo de vida del paciente y el de sus familiares.

Tabla 1. Cuidados preliminares ante sistemas de alarma

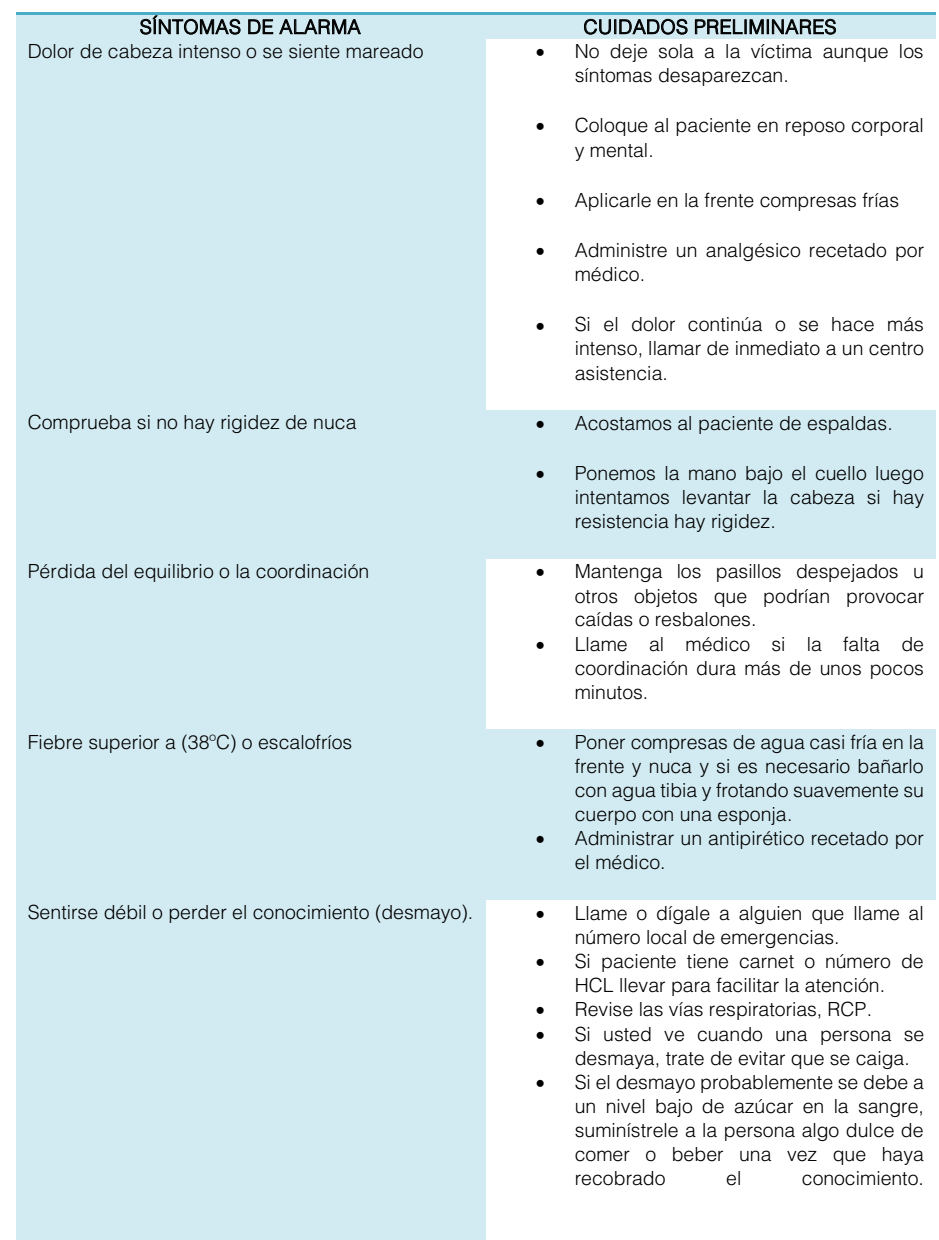

Fuente: (Changobalin, 2017) 


\section{Diagnóstico}

El cuadro clínico de sospecha típica incluye además náuseas, vómitos, dolor cervical, fotofobia y alteración de la conciencia. La valoración física puede revelar hemorragia retiniana, disminución del nivel de conciencia,meningismo y signos neurológicos localizadores (parálisis de pares craneales, hemiplejia o hemiparesia, trastornos del lenguaje, etc.). Los déficits focales neurológicos aparecen cuando el aneurisma presiona un nervio craneal, el sangramiento dentro del parénquima cerebral produce isquemia focal por vasoconstricción aguda después de la ruptura aneurismática. La rigidez de la nuca es provocada en respuesta inflamatoria de la sangre en el espacio subaracnoideo, puede tardar de tres a doce horas en aparecer o no presentarse en pacientes inconscientes con HSA menor. Las convulsiones son muy sugerente de HSA como causa de la cefalea, incluso aunque los pacientes hayan recuperado un nivel de conciencia normal.

Las manifestaciones en algunos pacientes pueden ser otros síntomas los más prominentesy no tener una cefalea severa. En ausencia de signos y síntomas clásicos, la HSA puede no diagnosticarse correctamente. No realizar una TC craneal o una punción lumbar (PL para descartar meningitis) cuando estaba indicado, además de malinterpretar sus resultados, explica la mayoría de errores en el diagnóstico.(Vermeulen \& Schull, 2007)(Edlow \& Caplan, 2000).

Los pacientes con un mal diagnostico tienden a estar menos enfermos y a tener una valoración neurológica normal, en tales casos, las complicaciones neurológicas ocurren más tarde,por este motivo, el 50\% de los pacientes tienen un riesgo mayor de muerte y discapacidadasociado al aneurisma cerebral(Kowalski, y otros, 2004).

Las dos clínicas que son usadas con más frecuencia, para evaluar la gravedad inicial de la HSA son las de Hunt-Hess y la WorldFederation of NeurologicalSurgeons
(WFNS); esta última es actualmente, la preferida en la mayoría de los servicios, en las unidades de cuidados intensivos. (Report of World Federation of Neurological Surgeons Committee on a Universal Subarachnoid Hemorrhage Grading Scale, 1988).

El diagnóstico puede ser muy difícil y pasar desapercibido cuando los síntomas son vagos. Entre las técnicas actuales para la evaluación,están basadas en estudios radiológicos, tales como arteriografía de contraste, tomografía computarizada, ultrasonido y resonancia magnética. Los estudios de imagen como la tomografía computarizada o el ultrasonido pueden ayudar rápidamente a confirmar o excluir el diagnóstico e identificar las complicaciones. Comúnmente los pacientes necesitan más de una prueba de imagen no invasiva para recabar la información necesaria para el diagnóstico. Un estudio reciente de 464 pacientes informó la angiografía por tomografía computarizada como la investigación inicial en el $61 \%$ de los casos, la ecocardiografía en el 33\%, la aortografía en el 4\% y la angiografía por resonancia magnética en el $2 \%$.

La tomografía computadorizada de cráneo (TC) elabora cortes horizontales de la cabeza, mostrando imágenes específicas del encéfalo que se utiliza para identificar la ubicación y el tipo de derrame cerebral que se ha producido.

Angiografía por tomografía computadorizada (angioTC) es una TC en combinación con la inyección de una solución de contraste intravenosa que se usa para ver las arterias del cerebro resaltadas y explorar los aneurismas.

Imágenes por resonancia magnética (IRM) forman imágenes ampliadas de estructuras del encéfalo con la finalidad de detectar cambios pequeños en el cerebro y ayudar a la localización y el diagnóstico de una hemorragia cerebral. 

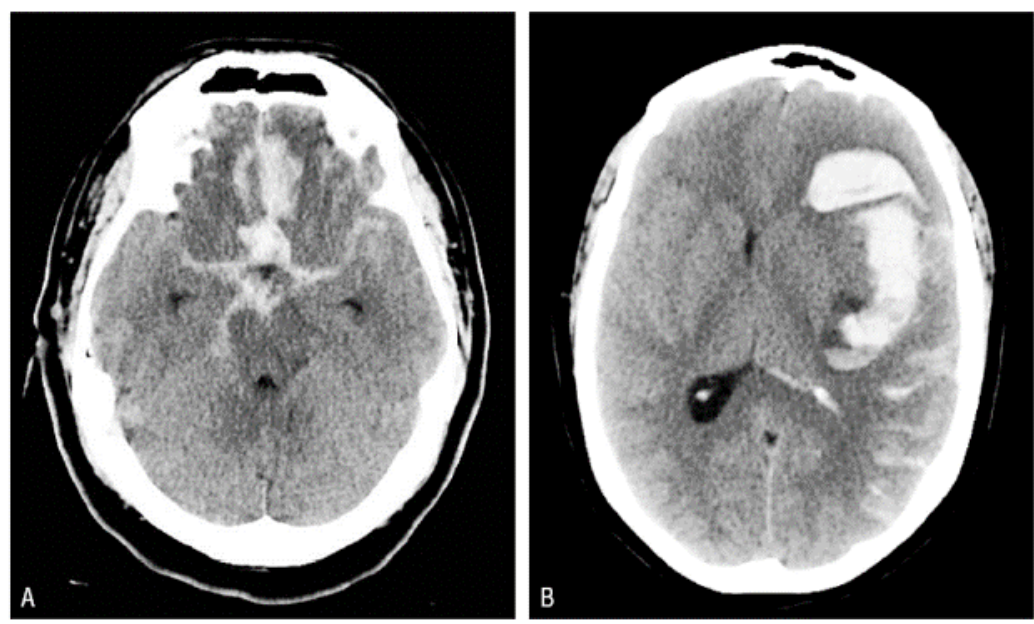

Gráfica 1. Caso A rotura de aneurisma; caso B HSA con hematoma

Fuente: (Guerrero, De La Linde, \& Pino Sánchez, 2008)

La angiografía por resonancia magnética (ARM) utiliza una combinación de tecnología de IRM y una solución de contraste intravenosa para visualizar los vasos sanguíneos del cerebro. El líquido de contraste hace que los vasos sanguíneos aparezcan opacos en la imagen de IRM, lo que permite que se visualice las arterias a evaluar. (FUCAC)

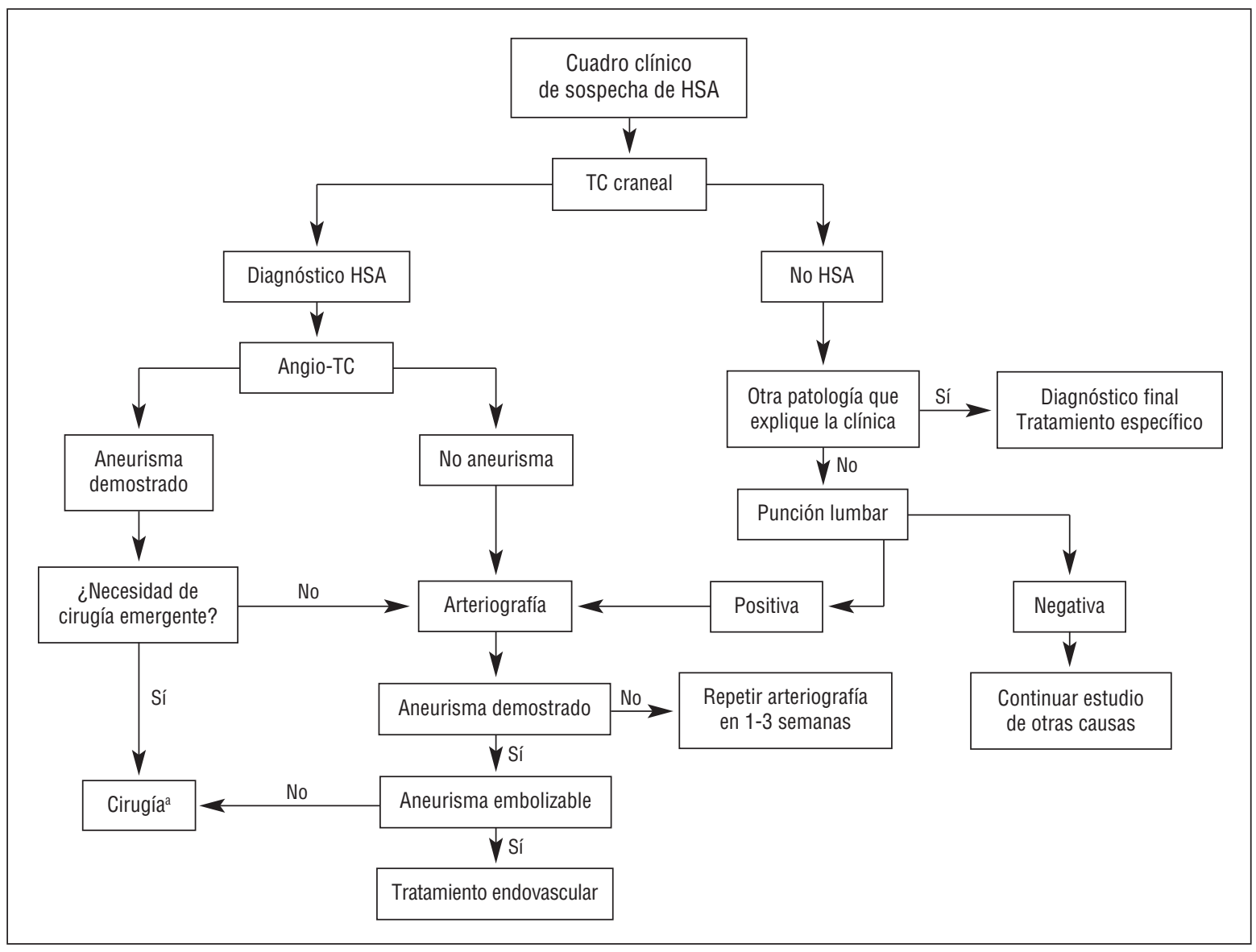

Gráfica 2. Diagnóstico Diferencial del Aneurisma Cerebral

Fuente: (Guerrero, De La Linde, \& Pino Sánchez, 2008) 


\section{Tratamiento}

Las decisiones sobre el tratamiento se basan en:

- Estado neurológico.

- Edad.

- Ubicación, tamaño y forma del aneurisma.

- Riesgo de ruptura del aneurisma.

- Disponibilidad de opciones de tratamiento.

- Aneurismas rotos frente a aneurismas no rotos.

De hecho, lo importante del tratamiento es evitar que sangre o vuelva a sangrar, de tal manera que, realizado cualquiera de los procedimientos estos no van a mejorar sus síntomas, salvo en el caso de aneurismas de gran tamaño que ponen presión contra los nervios. (The Brain Aneurysm Foundation, 2013).

Al tratar los aneurismas cerebrales, se definen dos opciones quirúrgicas donde ambas tienen algún riesgo para el paciente:

El clipadomicrovascular, este procedimiento se trata de cortar el flujo sanguíneo al aneurisma, bajo anestesia, se extrae una sección del cráneo y se localiza el aneurisma. El neurocirujano usa un microscopio para aislar el vaso sanguíneo que alimenta al aneurisma y coloca un clip pequeño y metálico similar a un broche, deteniendo el suministro sanguíneo. El clip se fija en el paciente evitando el riesgo de sangrado futuro. Luego se vuelve a colocar el trozo de cráneo y se cierra el cuero cabelludo.

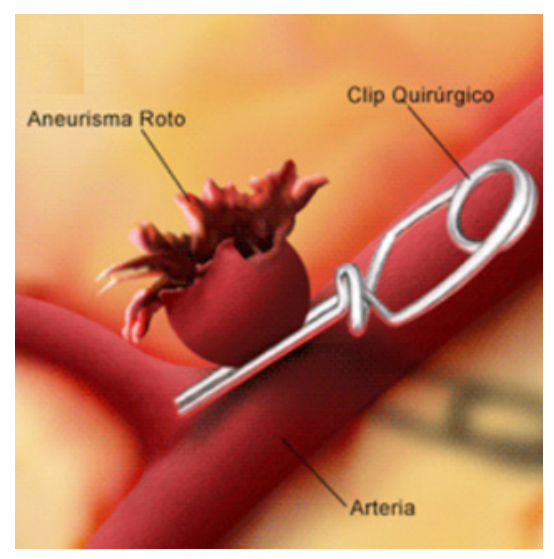

Gráfica 3. Clipado Microvascular

Fuente: (Guerrero, De La Linde, \& Pino Sánchez, 2008)

La embolizaciónendovascular,es una técnica de poca invasión, ya que, no requiere de una incisión en el cráneo. Este procedimiento se puede realizar con anestesia general o local, cuando el paciente es anestesiado, el neurocirujano introduce un tubo plástico hueco (un catéter) dentro de una arteria (generalmente en la ingle) y lo avanza usando angiografía por el cuerpo hasta el sitio del aneurisma. Utiliza un alambre guía, se pasan espirales removibles (espirales de alambre de platino) o pequeños balones de látex por el catéter y se liberan dentro del aneurisma. Los espirales o balones llenan el aneurisma, obstruyendo la circulación, y hacen que la sangre se coagule, lo que destruye eficazmente el aneurisma. 


\section{Gráfica 4. Embolización Endovascular}

Fuente: MAYO 2017

Prevención y Cuidados ante el diagnóstico de Aneurisma Cerebral:

No existen maneras de evitar el desarrollo de un aneurisma en el cerebro. Los pacientes diagnosticados deben controlar cuidadosamente la presión arterial y evitar situaciones de estrés que les suba la presión, dejar de fumar, y evitar el consumo de drogas estimulantes. (National Institute of Neurological, 2016)

Escala de riesgos de caídas para pacientes hospitalizados.

Las caídas se definen como eventos involuntarios que hacen perder el equilibrio y dar con el cuerpo en tierra u otra superficie firme que lo detenga.

Escala de Downton:

Si la puntuación es menor o igual de 2 el paciente no requiere cuidados específicos relacionados con el riesgo de caídas. Se ofrecen medidas sobre actividad física, prescripción de ejercicio y ajuste del entorno para optimizar su seguridad, si se observa algúncambio en su estado mental o físico se revalúa.

Si la puntuación Downton es mayor de 2 hay riesgo de caídas cuya disposición aumenta en sufrir daño físico. Se hace una evaluación de los posibles factores de riesgo y un plan de cuidados que contenga precauciones especiales.

Escala Morse:

Es una herramienta rápida y simple para valorar la posibilidad de que un paciente sufra una caída, se considera como "rápida y fácil de usar", considerando que, la calificación del riesgo lleva menos de 3 minutos. Seis variables son examinadas y con esto se confirma que tiene valor predictivo. Se utiliza tanto en los hospitales como en centros de internación de pacientes crónicos.

\section{Cuidados de Pacientes Intervenidos por Aneurisma Cerebral}

Actividades sugeridas para el deterioro de la movilidad(Changobalin, 2017), debido a la limitación del movimiento independiente, intencionado, del cuerpo, de una o más extremidades:

- Cambios de posición (dorsal, lateral, semifowler)

- Hidrate la piel para evitar que se reseque. (Lubriderm, aceite de benjuí)

- Ejercicios pasivos (flexión y extensión de las articulaciones como cabeza, mu-

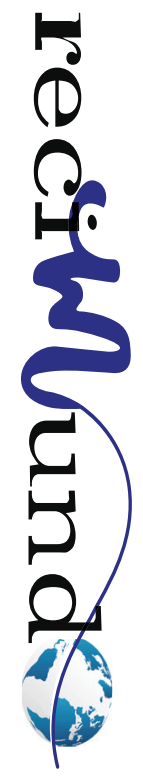


ñeca, codos, rodilla, dedos de los pies), realizado por el cuidador.

- Ejercicios activos según posibilidades del paciente (flexión, extensión, aducción, abducción y rotaciones).

Actividades sugeridas para el deterioro de la movilidad del cuello:

- Inclinación a la izquierda y derecha de la cabeza

- Rotación a la izquierda y derecha de la cabeza

- Flexión y extensión de la cabeza

Actividades sugeridas para el deterioro de la movilidad del brazo:

- Abducción del brazo

- Flexión del brazo

- Extensión del brazo

Actividades sugeridas para el deterioro de la movilidad de la mano:

- Puño cerrado.

- Extensión de muñeca.

- Reflejo tónico extensor del pulgar.

- Extensión semi-voluntaria de dedos. Alternar cierre y abertura.

- Extensión voluntaria de dedos.

Actividades sugeridas para el deterioro de la movilidad en la rodilla:

- Flexión de la rodilla.

- Extensión de la rodilla.

Actividades sugeridas para el deterioro de la movilidad del pie:

- Flexión o extensión plantar y dorsal

- Supinación y pronación

- Abducción y Aducción

- Utilice calzado cómodo para la deam- bulación.(casual, deportiva )

- Siéntese en un lado de la cama a 90( posiciónsemifowler) antes de iniciar la deambulación.

- Utilice un bastón, muletas, silla de ruedas como apoyo.

- Camine a paso lento por la habitación a intervalos regulares (mañana, tarde, noche)

- Guie al paciente en la realización de ejercicios y actividades para mejorar la coordinación y el equilibrio.

Actividades sugeridas para el manejo de la ansiedad:

- Consiste en un estado de tensión física o psicológica, que ocurre ante los inconvenientes de la vida diaria y ante temores reales o imaginarios, que cuando, adquiere proporciones exageradas y afecta de manera negativa la vida

- Respire lenta y profundamente durante unos minutos.

- Practica ejercicios relajación en un lugar tranquilo, silencioso y con temperatura agradable.

- Escuche música que lo tranquilice.

- Aumente la vida social, vincularse en reuniones informales y cultive la amistad.

- Hable con un amigo o un ser querido de sus sentimientos y mensajes no expresados

Actividades sugeridas para el insomnio en el paciente:

- Es el trastorno de sueño más frecuente en la población general. Consiste en una reducción de la capacidad para dormir.

- Crea un ambiente tranquilo (luz, temperatura, colchón cama) para favorecer el sueño

- Lleve a cabo medidas agradables (ma- 
saje, contacto afectuoso)

- Controle y evite ruidos indeseados o excesivos en lo posible (radio, televisión, alarma de reloj)

- Establezca una hora adecuada para (despertar y acostarse)

- Evite consumir bebidas que contengan cafeína antes de dormir. (café)

Actividades sugeridas para los ejercicios físicos terapéutico, con la finalidad de mejorar la capacidad de moverse, determinando el grado de independencia del paciente(el aseo, vestido, comida), por tal motivo se sugiere:

\section{Realice ejercicios de resistencia}

- Ejecutar por lo menos 30 minutos movimientos que le ayude a acelerar la respiración (caminar)

- Ejercicios de 30 minutos al día.

\section{Realice ejercicios de fortalecimiento}

- Aumente la resistencia muscular (pesas)

- Levantarse solo de la silla sin apoyo de las manos ni los brazos.

- Intente pararse en un pie y luego en el otro de ser posible

- Camine en línea recta con un pie adelante del otro.

\section{Ejercicios de estiramiento}

- Moverse con más facilidad le ayudara hacer cosas como agacharse para amarrarse los zapatos o toparse las puntas de los pies.

\section{Conclusion}

Los aneurismas pueden desarrollarse y permanecer asintomáticos. Comúnmente, si se detectan y se tratan puede evitarse su rotura. Para su diagnóstico se usan pruebas de imágenes, siendo las más utilizadas la tomografía computada y la ecografía. Frecuente- mente, su detección es incidental durante pruebas hechas por otras razones o como hallazgo de autopsia. El conocimiento de la patología de estos trastornos vasculares ha permitido el desarrollo de nuevas formas de terapia menos invasivas para el manejo de los pacientes. Los aneurismas son trastornos de la pared vascular que deben tenerse en cuenta, siempre como un diagnóstico diferencial en pacientes altamente sospechosos o en personas previamente sanas que presentan muerte súbita, razón por la cual, son de importancia en el ámbito de la medicina forense.

La resonancia magnética, permite un diagnóstico más acertado que la tomografía, sin embargo, mientras que un estudio de TC cerebral puede obtenerse de 8 a 10 minutos, en la RM, suele tardar alrededor de 20 minutos en hacer la adquisición de las imágenes utilizando las secuencias adecuadas, por lo que, ante los síntomas de alarma la velocidad del diagnóstico y tratamiento, disminuye las secuelas de esta patología en el paciente.

Para la persona, la familia y la comunidad es importante pues favorece el cuidado integral de manera individualizada y al profesional de Enfermería le favorece en la toma de decisiones y la evaluación crítica y analítica de las acciones realizadas, al igual que, dichas acciones con el único fin de mantener o recuperar el bienestar físico, mental y social de los individuos que tiene bajo su cuidado.

Finalmente, la información detalla en cada una de las actividades que el paciente debe ejecutar en compañía de un familiar, estas tareas van desde la identificación oportuna de signos de alarma, deterioro de la movilidad, ansiedad, insomnio y a su vez les permitirá estar en la capacidad de resolver posibles problemas que se presenten, proporcionando una atención de calidad en beneficio de una pronta recuperación del paciente. 
En conclusión, este trabajo ha sido el realizar una guía en el buen manejo de las medidas generales y específicas que requiere un paciente con aneurisma cerebral por medio de craneotomía para la prevención y disminución de complicaciones.

\section{Bibliografía}

Broderick, J., Sauerbeck, R., Foroud, T., \& ét al. (2005). The Familial Intracranial Aneurysma (FIA) study protocol (Vol. 6). Bmc Medical Genetics. Recuperado el Jul de 2010, de http://www.biomedcentral.com/1471-2350/6/17/

Carmona Simarro, J., Gallego Lopez , J., \& LLabata Carabal, P. (2005). Paciente neurocritico. Actuación integral de Enfermeria (Vol. 6). Med clínica - Enfermeria Global. Obtenido de http://revistas. um.es/eglobal/article/viewFile/502/499

Changobalin, J. (Junio de 2017). Aneurisma cerebral y su influencia en la calidad de vida. Ambato, Ecuador: Universidad Técnica de Ambato.

Edlow, J., \& Caplan, L. (2000). Avoiding pitfalls in the diagnosis of subarachnoid hemorrhage (Vol. 42). N Engl J Med.

FUCAC. (S.F.). Fundacion para la cobertura del Aneurisma Cerebral. Obtenido de http://fucac. org/aneurisma-cerebral/diagnostico-del-aneurisma-cerebral/

García, A., \& Pardo, G. (s.f.). Cirugia Tomo IV. Cuba: Ciencias Médicas.

Guerrero , F., De La Linde, C., \& Pino Sánchez, F. (2008). Manejo general en Cuidados Intensivos del paciente con hemorragia subaracnoidea espontánea (Vol. 32). Granada: Hospital Universitario Virgen de las Nieves.

(2008). Guidelines for the Surgical Treatment of Unruptured Intracranial Aneurysms. The First Annual.

Harrod, C., Bendok, B., Batjer, H., \& ét al. (2005). Presenting with Aneurysmal Subarachnoid Hemorrhage Prediction of Cerebral Vasospasm in Patients (Vol. 56). Neurosurgery. Recuperado el Julio de 2010, de http://www.ncbi.nlm.nih.gov/ pubmed/15792502

INEC. (2015). Instituto Nacional de Estadísticas y Censos. Ecuador.

Kowalski, R., Claassen, J., Kreiter, K., Bates, J., Ostap, K., Vich, N., ... et al. (2004). Initial misdiagnosis and outcome after subarachnoid hemorrhage (Vol. 291). JAMA.
Lauric, A., Miller , E., Frisken, S., \& ét al. (2010). Automated detection of intracranial aneurysms based on parent vessel 3D analysis (Vol. 14). Medical Image Analysis.

Mayo Clinic. (2017). Recuperado el 22 de Nov de 2017, de https://www.mayoclinic.org/es-es/diseases-conditions/brain-aneurysm/symptoms-causes/ syc-20361483

Mocco, J., Zacharia, B., Komotar, R., \& ét al. (2006). A review of current and future medical therapies for (Vol. 21). Neurosurgery. Recuperado el Jul de 2010, de http://thejns.org/doi/pdf/10.3171/ foc.2006.21.3.9

National Institute of Neurological. (2016). National Institute of Neurological Disorders and Stroke. Recuperado el 20 de Dic de 2016, de https://espanol. ninds.nih.gov/trastornos/aneurismas_cerebrales. $\mathrm{htm}$

OMS. (2015). Informe anual Organizacion Mundial de la Salud.

Report of World Federation of Neurological Surgeons Committee on a Universal Subarachnoid Hemorrhage Grading Scale (Vol. 68). (1988). J Neurosurg.

The Brain Aneurysm Foundation. (2013). Introduccion a los aneurismas cerebrales y sus tratamientos. Obtenido de https://www.bafound.org/wp-content/ uploads/2016/01/2013-Spanish-BAF_Intro_Treatment_booklet_Final_ES-US_0.pdf

Vermeulen, M., \& Schull, M. (2007). Missed diagnosis of subarachnoid hemorrhage in the emergency department (Vol. 38). Stroke. 


\section{CITAR ESTE ARTICULO:}

Santana Bailón, E., Cevallos Vega, D., Moyano Vega, C., Burgos Delgado, R., Arteaga Castro, Y., \& Durán González, J. (2020). Prevención y cuidado de los pacientes diagnosticados con aneurisma cerebral. RECIMUNDO, 4(1), 403415. doi:10.26820/recimundo/4.(1).enero.2020.403-415

\section{(c) (1) $\circledast(0$ \\ BY NC SA}

RECONOCIMIENTO-NOCOMERCIAL-COMPARTIRIGUAL CC BY-NC-SA

CC BY-NC-SA
ESTA LICENCIA PERMITE A OTROS ENTREMEZCLAR, AJUSTARY ESTA LICENCIA PERMITE A OTROS ENTREMEZCLAR, AJUSTAR Y
CONSTRUIR A PARTIR DE SU OBRA CON FINES NO COMERCIALES, SIEMPRE Y CUANDO LE RECONOZCAN LA AUTORÍA Y SUS NUEVAS CREACIONES ESTÉN BAJO UNA LICENCIA CON LOS MISMOS TÉRMINOS. 